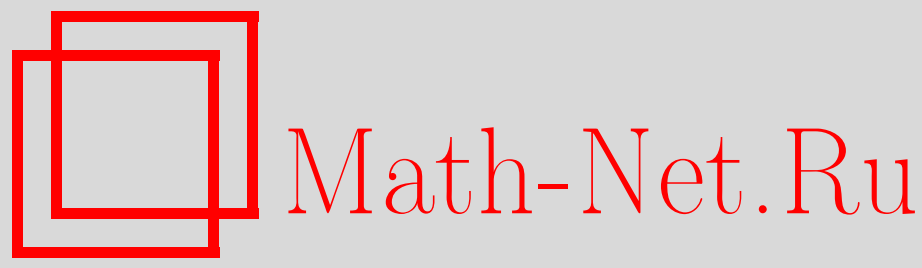

Ф. М. Малышев, О доле матриц с максимальной аддитивной сложностью, Дискрет. матем., 2014, том 26, выпуск $3,76-78$

DOI: https://doi.org/10.4213/dm1291

Использование Общероссийского математического портала Math-Net.Ru подразумевает, что вы прочитали и согласны с пользовательским соглашением http://www. mathnet.ru/rus/agreement

Параметры загрузки:

IP : 3.89 .185 .249

26 апреля 2023 г., 07:57:14

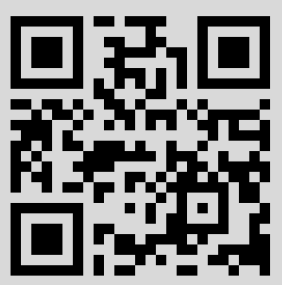




\title{
О доле матриц с максимальной аддитивной сЛОЖНОСТЬЮ
}

\author{
() 2014 г. . М. Малышев*
}

\begin{abstract}
Под аддитивной сложностью невырожденной матрицы размера $n$ в статье понимается минимальное число сложений в цепочке элементарных преобразований над строками, приводящей матрицу к единичной. Доказывается, что с ростом порядка поля почти все матрицы обладают максимально возможной аддитивной сложностью, равной $(n-1) n$. Показано, что матрицы аддитивной сложности $(n-1) n$ являются $M D S$-матрицами.
\end{abstract}

Ключевые слова: сложность матриц, аддитинвная сложность, $M D S$-матрицы.

Каждая невырожденная матрица $L \in G L(n, K)$ размера $n \geqslant 1$ над произвольным полем $K$ может быть представлена в виде произведения матриц трёх следующих типов: 1) подстановочных, 2) диагональных, 3) матриц с единицами по главной диагонали и нулевыми остальными элементами за исключением какого-то одного, равного единице. Следуя традиции, назовём эти матрицы элементарными.

Умножение матрицы $X \in G L(n, K)$ на элементарные матрицы слева отвечает преобразованиям над строками матрицы $X$, соответственно: перестановке строк, умножению строк на ненулевые элементы поля, прибавление к одной строке какой-то другой строки, остающейся неизменной.

Для некоторых приложений представляют интерес матрицы, для которых в рассматриваемых произведениях число сомножителей типа 3) имеет гарантированную нижнюю оценку.

Определение 1. Аддитивной сложностью $\vartheta_{L}$ матрицы $L \in G L(n, K)$ назовём минимальное число элементарных матриц типа 3 ) в представлениях $L$ произведениями элементарных матриц.

Нетрудно понять, что $0 \leqslant \vartheta_{L} \leqslant(n-1) n$. Для произведений подстановочных матриц с диагональными и только для них аддитивная сложность равна нулю. Верхняя оценка величины $\vartheta_{L}$ следует из метода Гаусса сведения произвольной невырожденной матрицы к единичной матрице. Но не при всяких $n$ и $K$ имеются невырожденные матрицы аддитивной сложности $(n-1) n$.

Определение 2. Матрицу $L \in G L(n, K)$ называем предельно аддитивно сложной, если $\vartheta_{L}=\max _{\mathcal{L} \in G L(n, K)} \vartheta_{\mathcal{L}}$, и максимально аддитивно сложной, если $\vartheta_{L}=(n-1) n$.

* Место работы: Математический институт им. В.А. Стеклова РАН, e-mail: malyshevfm@mi.ras.ru 
Для поля $K=G F(2)$ при всех $n>1$ максимально аддитивно сложных матриц не существует.

При $n=2$ имеем $\vartheta_{L}=1$ для матриц $L=\left(\begin{array}{ll}a & b \\ c & d\end{array}\right)$ с одним нулевым элементом, и $\vartheta_{L}=2$ для матриц $L$ без нулевых элементов. Таким образом, при $n=2$ максимально аддитивно сложные матрицы существуют только при $|K|>2$, а всё множество максимально аддитивно сложных матриц совпадает с множеством так называемых $M D S$-матриц (см. [1]). В общем случае такого совпадения нет. В конце статьи будет построено семейство $M D S$-матриц для $n=3$, которые не являются максимально аддитивно сложными.

Напомним [1], что $M D S$-матрицей является такая матрица размера $n \times n$, у которой все $\sum_{i=1}^{n}\left(\begin{array}{c}n \\ i\end{array}\right)^{2}=\left(\begin{array}{c}2 n \\ n\end{array}\right)-1$ подматриц на пересечении $i$ строк и $i$ столбцов, $i=1, \ldots, n$, являются невырожденными.

Далее будут доказаны две следующие теоремы.

Теорема 1. При каждом $n \geqslant 1$ доля максимально аддитивно сложных матрич 6 множестве всех матрии размера $n \times n$ стремится $\kappa$ единице при неограниченном увеличении порядка поля $K$.

Теорема 2. Каждая максимально аддитивно сложная матрица является MDS-матричей.

Доказательство теоремы 1. Обозначим $N_{\vartheta}=\left|\mathcal{N}_{\vartheta}\right|$, где $\mathcal{N}_{\vartheta}=$ $\left\{L \in G L(n, K) \mid \vartheta_{L} \leqslant \vartheta\right\}, 0 \leqslant \vartheta \leqslant(n-1) n$. Индукцией по $\vartheta$ докажем, что

$$
N_{\vartheta} \leqslant c_{n} \cdot k^{n+\vartheta}
$$

где $k=|K|-1$, а $c_{n}$ - константа, зависящая от $n$. В качестве $c_{n}$ можно взять число последовательностей длины не более $2(n-1) n+1$ из чередующихся матриц типа 2$)$ и 3). Это число не зависит от поля $K$.

Множество $\mathcal{N}_{\vartheta}$ разобьём на классы эквивалентности, считая эквивалентными матрицы, получающиеся одна из другой умножением слева на диагональную матрицу. В одном классе эквивалентности $k^{n}$ матриц. Один класс эквивалентности задаётся последовательностью $\left(\hat{l}_{1}, \ldots, \hat{l}_{n}\right)$ из $n$ различных точек $(n-1)$-мерного проективного пространства $P K^{n}$. Одна точка $\hat{l}_{i} \in P K^{n}, i=1, \ldots, n$, задаёт с точностью до ненулевого множителя $i$-ю строку $l_{i} \in K^{n}$ матрицы $L$.

Вместо неравенства (1) достаточно доказать неравенство

$$
M_{\vartheta} \leqslant c_{n} \cdot k^{\vartheta},
$$

где $M_{\vartheta}=N_{\vartheta} / k^{n}$ - число классов эквивалентности матриц $L \in G L(n, K)$ с $\vartheta_{L} \leqslant \vartheta$.

Для конкретной последовательности длиной $2 \vartheta+1$ из чередующихся подстановочных матриц числом $\vartheta+1$ и матриц типа 3$)$ числом $\vartheta$ через $\mathcal{R}_{\vartheta}$ обозначим множество матриц, являющихся произведениями этих $2 \vartheta+1$ матриц и $\vartheta+1$ произвольных диагональных матриц, располагающихся в начале и в конце последовательности и по одной между матрицами типа 3 ). Через $r_{\vartheta}$ обозначим число классов эквивалентности в $\mathcal{R}_{\vartheta}$.

Индукцией по $\vartheta$ докажем неравенство

$$
r_{\vartheta} \leqslant k^{\vartheta}
$$


из которого будет следовать неравенство (2), а значит, и неравенство (1).

Для $\vartheta=0$ неравенство (3) справедливо. Индуктивный переход следует из того, что преобразование типа 3), например, прибавление $i$-й строки к $j$-й, ко всем матрицам одного класса эквивалентности $\left(\hat{l}_{1}, \ldots, \hat{l}_{n}\right)$, будет предоставлять матрицы ровно из $k$ классов эквивалентности, конкретно, из $\left(\hat{l}_{1}, \ldots, \hat{l}_{j-1}, \hat{l}, \hat{l}_{j+1}, \ldots, \hat{l}_{n}\right)$, где $\hat{l}$ пробегает точки проективной прямой, проходящей через $\hat{l}_{i}$ и $\hat{l}_{j}$, за исключением самих этих точек. После того, как неравенство (1) доказано, имеем $N_{(n-1) n-1} \leqslant$ $c_{n} \cdot k^{n+(n-1) n-1}=c_{n} \cdot k^{n^{2}-1}$. Теперь для доказательства теоремы 1 остаётся заметить, что доля невырожденных матриц в множестве всех $(k+1)^{n^{2}}$ матриц размера $n \times n$ стремится к единице при неограниченном увеличении порядка поля $K$. Этот хорошо известный факт проверяется индукцией по $n$. Теорема 1 доказана.

Доказательство теоремы 2 проведём от противного. Пусть в максимально аддитивно сложной матрице $L \in G L(n, K)$ определитель подматрицы $L_{0}$ на пересечении (для определённости) $r$ первых строк и $r$ первых столбцов нулевой, $1 \leqslant r<n$, и последняя $r$-я строка подматрицы $L_{0}$ является линейной комбинацией предыдущих её $r-1$ строк. После применения элементарных преобразований над строками с числом сложений не более $r-1$, последняя строка в $L_{0}$ будет нулевой. Затем, прибегая к методу Гаусса для первых $r$ столбцов, в этих столбцах будет по одному ненулевому элементу после применения элементарных преобразований не более чем с $(n-2) r$ сложениями строк. Наконец, того же добиваемся в последних $n-r$ столбцах после привлечения элементарных преобразований не более чем с $(n-1)(n-r)$ сложениями строк. Общее число затрачиваемых сложений строк будет ограничиваться сверху величиной $r-1+(n-2) r+(n-1)(n-r)=(n-1) n-1<(n-1) n$. Это противоречит максимальной аддитивной сложности матрицы $L$. Теорема 2 доказана.

Примером 4-параметрического семейства $M D S$-матриц при $n=3$, которые не являются максимально аддитивно сложными, служит

$$
\mathcal{L}_{0}=\left(\begin{array}{ccc}
1+x y & c+y+c x y & y \\
x y & y+c x y & y \\
x+a+a x y & c x+a c+a y+a c x y & 1+a y
\end{array}\right),
$$

где значения параметров $a, c, x, y \in K^{*}=K \backslash\{0\}$ должны быть такими, чтобы все 9 элементов матрицы $\mathcal{L}_{0}$ были ненулевыми. Невырожденность всех 9 подматриц в $\mathcal{L}_{0}$ размера $2 \times 2$ дополнительно потребует условий: $a+x \neq 0, a c \neq 1, c+y \neq 0$. Матрица $\mathcal{L}_{0}$ получается из единичной матрицы в результате последовательности операций: $B_{1}\left(c^{-1}\right), B_{3}\left(x^{-1}\right), A_{21}, B_{1}(c), A_{13}, B_{3}(x), A_{32}, B_{2}(y), A_{21}, B_{1}(a), A_{13}, B_{1}\left(a^{-1}\right)$, где $A_{i j}$ - прибавление $i$-й строки к $j$-й, $B_{i}(\alpha)$ - умножение $i$-й строки на $\alpha$. Таким образом, $\vartheta_{\mathcal{L}_{0}} \leqslant 5<6$. При достаточно большой мощности поля это семейство непусто.

Автор благодарен А.М. Зубкову за оказанную помощь в подготовке статьи и критические замечания к первому её варианту.

\section{Список литературы}

1. Мак-Вильямс Ф.Д., Слоэн Н.Д.А., Теория кодов, исправляющих ошибки, М.: Связь, 1979 . 